\title{
NITROGEN AVAILABILITY IMPROVES THE PHYSIOLOGICAL RESILIENCE OF CORAL ENDOSYMBIONT CLADOCOPIUM GOREAUI TO HIGH TEMPERATURE ${ }^{1}$
}

\author{
Zhi Zhou (iD ${ }^{2}$ \\ Hainan Aquaculture Breeding Engineering Research Center, College of Marine Sciences, Hainan University, Haikou, Hainan \\ 570228, China \\ Department of Marine Sciences, University of Connecticut, Groton, Connecticut 06340, USA
}

Kaidian Zhang

Department of Marine Sciences, University of Connecticut, Groton, Connecticut 06340, USA

State Key Laboratory of Marine Environmental Science, Xiamen University, Xiamen, Fujian 361102, China

$$
\text { Lingui Wang, Yilu Su }
$$

Hainan Aquaculture Breeding Engineering Research Center, College of Marine Sciences, Hainan University, Haikou, Hainan 570228, China

Jierui Wang

State Key Laboratory of Marine Environmental Science, Xiamen University, Xiamen, Fujian 361102, China

$$
\text { Tingting Song }
$$

Hainan Aquaculture Breeding Engineering Research Center, College of Marine Sciences, Hainan University, Haikou, Hainan 570228, China

Xiaohong Yang

State Key Laboratory of Marine Environmental Science, Xiamen University, Xiamen, Fujian 361102, China

$$
\text { Jia Tang }
$$

Key Laboratory of Coastal Zone Environmental Processes, Yantai Institute of Coastal Zone Research, Chinese Academy of Sciences, Yantai, Shandong 264003, China

$$
\text { and Senjie Lin (iD) }{ }^{2}
$$

Department of Marine Sciences, University of Connecticut, Groton, Connecticut 06340, USA

State Key Laboratory of Marine Environmental Science, Xiamen University, Xiamen, Fujian 361102, China

The physiological response of symbiotic Symbiodiniaceae to high temperature is believed to result in coral bleaching. However, the potential effect of nitrogen availability on heat acclimatization of symbiotic Symbiodiniaceae is still unclear. In this study, physiological responses of Symbiodiniaceae Cladocopium goreaui to temperature and nitrogen nutrient stress conditions were investigated. Nitrogen deficiency caused significant declines in cell concentration and chlorophyll content per cell, but significant increases in nitric oxide synthase activity, caspase 3 activation level, and cellular carbon content of $C$. goreaui at normal temperature. Algal cells under high temperature and

\footnotetext{
${ }^{1}$ Received 28 August 2020. Accepted 8 January 2021.

${ }^{2}$ Author for correspondence: e-mails zhouzhi@hainanu.edu.cn; senjie.lin@uconn.edu.

Zhi Zhou and Kaidian Zhang contributed equally to this work.

Editorial Responsibility: T. Mock (Associate Editor)
}

nitrogen deficiency showed significant rises in $\mathbf{F v}$ / Fm, catalase activity, and caspase 3 activation level, but no significant changes in cell yield, cell size, chlorophyll content, superoxide dismutase, nitric oxide synthase activity, and cellular contents of nitrogen and carbon, in comparison with those under normal temperature and nitrogen deficiency. Growth, chlorophyll, and nitrogen contents of algal cells under the high temperature and nitrogenreplete conditions were significantly higher than those under high temperature or nitrogen deficiency alone, whereas nitric oxide synthase activity, superoxide dismutase activity, catalase activity, carbon content, and caspase 3 activation level exhibited opposite trends of variation. Transcriptomic and network analyses revealed ion transport and metabolic processes mainly involved in regulating these physiological activities under different temperature and nitrogen nutrient. The 
totality of results shows that high temperature activates stress responses, induces antioxidant capacity of apoptosis, and limits the growth rate of C. goreaui. Adequate nitrogen nutrient can improve the resilience of this Symbiodiniaceae against heat stress through repressed apoptosis, promoted ion transport, and optimized metabolism.

Key index words: apoptosis; heat stress; nitrogen limitation; stress response; symbiodiniaceae

Abbreviations: CAT, catalase; DEGs, differentially expressed genes; FPKM, Fragments Per Kilobase of exon per Million fragments mapped; GO, gene ontology; Heat_N-, heat stress and $\mathrm{N}$-deficient group; Heat_N+, heat stress and $\mathrm{N}$-supplemented group; L1-N, L1 medium without $\mathrm{N}$-nutrient addition; $\mathrm{N}-, \mathrm{N}$-deficient group; $\mathrm{N}+, \mathrm{N}$-supplemented group; N-nutrient, nitrogen nutrient; NOS, nitric oxide synthase; $\mathrm{Nr}$, non-redundant protein database; PBS, phosphate-buffered saline; SMART, Simple Modular Architecture Research Tool; SOD, superoxide dismutase; SRA, Short Read Archive

Dinoflagellates of the family Symbiodiniaceae are the most prevalent photosynthetic microalgae in tropical and subtropical coral reef ecosystems and provide energy and organic carbon to support the growth of scleractinian corals and the function of the reef ecosystems (Rosenberg et al. 2007). Phylogenetic reconstruction, genomic comparison, and morphological characterization have indicated that the family Symbiodiniaceae is composed of diverse lineages, including genera of Symbiodinium (Clade A), Breviolum (Clade B), Cladocopium (Clade C), Durusdinium (Clade D), Effrenium (Clade E), Fugacium (Clade F), and Gerakladium (Clade G; LaJeunesse et al. 2018). These genera except Effrenium contain species able to live symbiotically with many invertebrates, notably scleractinian coral, and the mutualisms are fundamental to the existence of coral reef ecosystems worldwide (Houlbreque and Ferrier-Pages 2009). These symbionts provide up to $95 \%$ of their coral hosts' energy requirements by transferring photosynthetically fixed carbon to the coral (Falkowski et al. 1984, GonzalezPech et al. 2019).

The symbiotic association of scleractinian coral and Symbiodiniaceae commonly flourishes in nutrient-poor environments, mainly by nutrient recycling within the symbiotic system (Radecker et al. 2015). The symbiotic association takes up and assimilates extremely limited nutrients, especially nitrogen, which is needed as a building block of nucleic acids and proteins, from the ambient seawater to sustain their growth (Tanaka et al. 2018). Within the symbiotic association, the coral host provides soluble inorganic nitrogen such as ammonium for its symbiont as the primary nitrogen source, in exchange for photosynthates (Shinzato et al. 2011, Pernice et al.
2012). Under inadequate nitrogen supply to the holobiont, symbiodiniacean symbionts usually experience nitrogen limitation since they require more nitrogen than does their coral host (Peng et al. 2012). It is believed that some level of nitrogen limitation in the symbionts is beneficial for the maintenance of the symbiotic relationship because it causes the symbionts to produce and translocate more photosynthetic carbon products and while consuming less for their own proliferation (Radecker et al. 2015, Morris et al. 2019). Therefore, the degree of nitrogen limitation can be critical to the symbiotic relationship's stability (Morris et al. 2019). The role of nitrogen nutrients in the physiological performance of Symbiodiniaceae under global climate and seawater warming, the primary cause of coral bleaching, is still poorly understood, however.

The question about nutrients and seawater warming is important, particularly because global warming, largely due to greenhouse gas released by humans, is accompanied by coastal eutrophication, another consequence of human activities. Against this backdrop are the frequent and severe bleaching episodes of scleractinian coral over the past decades (Vidal-Dupiol et al. 2011). Heat bleaching of scleractinian coral could result from excessive oxidative pressure owing to increased reactive oxygen species and damaged D1 protein in symbiotic Symbiodiniaceae (Lesser 1997, Downs et al. 2002). Nitrogen enrichment in the environment can raise the symbionts' reproduction rate, and symbiont overpopulation can result in coral bleaching through induced respiratory burst and apoptosis (Lin et al. 2017, Yuan et al. 2017). Furthermore, there are potentially interactive effects of seawater warming and nitrogen enrichment on scleractinian coral. However, results documented to date are inconsistent, some showing increases and others decreases in the susceptibility of scleractinian corals to heat bleaching under elevated temperature and nitrogen availability (Wiedenmann et al. 2012, Zhou et al. 2017, Hall et al. 2018).

In this study, we used Cladocopium goreaui as a representative Symbiodiniaceae species to investigate the effect of temperature and nitrogen availability on Symbiodiniaceae. Cladocopium goreaui is a Symbiodiniaceae species that belongs to clade $\mathrm{C}$, the most abundant clade of Symbiodiniaceae in coral reef ecosystems in the Pacific. To understand the potential effects of nitrogen availability on this species' heat resistance, its growth and photosynthesis parameters, antioxidative and apoptotic enzyme activities, cellular nitrogen and carbon contents, and transcriptome profiles were analyzed under stress treatments with two temperature and two nitrogen conditions. Our results provide fundamental insights for predicting how coral reefs will cope with the pressure of global warming and eutrophication in the future ocean. 


\section{MATERIALS AND METHODS}

Algal culture and experimental treatment. C. goreaui strain CCMP 2466 (Clade C1), originally isolated from the anemone Discosoma sanctithomae, was provided by the National Center for Marine Algae and Microbiota (Bigelow Laboratory for Ocean Sciences, East Boothbay, Maine, USA). Cells were cultured in sterilized oceanic seawater (from oligotrophic sea area, nitrogen concentration $0.5-1.5 \mu \mathrm{mol} \cdot \mathrm{L}^{-1}$ ), which was filtered through $0.22-\mu \mathrm{m}$ membranes, enriched with the full nutrient regime of the L1 medium (Su et al. 2020). Control cultures were incubated at $26^{\circ} \mathrm{C}$ and under a 14:10 h light night cycle, with a photon flux of $110 \mu \mathrm{mol}$ photons $\cdot \mathrm{m}^{-2} \cdot \mathrm{s}^{-1}$.

For nitrogen nutrient $(\mathrm{N})$ treatment, a normal culture was grown until it reached the exponential growth phase, and then, it was inoculated into the fresh L1 medium without $\mathrm{N}$ addition (L1-N; i.e., the low concentration of $\mathrm{N}$ in the oceanic seawater was the only $\mathrm{N}$ available). When the culture stopped growing, this culture was used as inoculum to set up four different treatments, with the same initial concentration of $6.89 \pm 0.08 \times 10^{4}$ cells $\cdot \mathrm{mL}^{-1}$ : (i) $26^{\circ} \mathrm{C}$ with L1-N medium as $\mathrm{N}$-deficient group $\left(\mathrm{N}-\right.$, control), (ii) $26^{\circ} \mathrm{C}$ with the $\mathrm{L} 1$ medium as $\mathrm{N}$-supplemented group $(\mathrm{N}+)$, (iii) elevated temperature at $32^{\circ} \mathrm{C}$ with $\mathrm{L} 1-\mathrm{N}$ medium as heat stress and $\mathrm{N}$-deficient group (Heat_N-), and (iv) elevated temperature at $32^{\circ} \mathrm{C}$ with $\mathrm{L} 1$ medium as heat stress and N-supplemented group (Heat_N+). For the heat stress groups, temperature was gradually increased by a rate of $1^{\circ} \mathrm{C}$ per $12 \mathrm{~h}$. All the groups were carried out with five replicates. Each culture was sampled at $24,48,72$, and $84 \mathrm{~h}$ after the onset of the treatment to determine the cell concentration, cell size, and enzyme activities. Samples at $84 \mathrm{~h}$ were also subjected to measurements of photosynthesis parameters, cellular carbon and nitrogen contents, and transcriptome profiling.

Measurement of cell concentration, cell size, photochemical effciency, and chlorophyll a content. Cell concentration was determined by direct counting using a Sedgwick-Rafter counting chamber under the microscope, and growth curves were plotted to depict growth phases (Lin et al. 2012). The average cell size was measured as equivalent spherical diameter using Z2-Coulter Particle Counter (Beckman Coulter, Indianapolis, IN, USA; Li et al. 2016). Photochemical efficiency was measured using Xe-PAM (Walz, Effeltrich, Germany) after the samples had been incubated in the dark for 15-30 min.

A 5-mL sample was filtered using a $25 \mathrm{~mm}$ Whatman $\mathrm{GF} / \mathrm{F}$ glass microfiber filter (GE Healthcare, Little Chalfont, UK) to obtain the cells. The algae-containing filter was immersed in $90 \%$ acetone and then incubated in the dark for $48 \mathrm{~h}$ at $4^{\circ} \mathrm{C}$ to extract Chlorophyll $a$, which was determined by using Turner Trilogy fluorometer (Turner Designs, San Jose, CA, USA) according to the non-acidification method (Arar and Collins 1997) and averaged to per cell content.

Determination of antioxidative enzyme activities and caspase 3 activation level. Algal cells were harvested from a $10 \mathrm{~mL}$ sample by centrifugation at $5,000 \mathrm{~g} 4^{\circ} \mathrm{C}$ for $10 \mathrm{~min}$. Cells pellets were resuspended in $1 \times$ PBS (phosphate-buffered saline, $\mathrm{pH}$ 7.4) and homogenized using glass beads $(0.5 \mathrm{~mm}$ diameter ceramic beads), and a bead-beater (FastPrep ${ }^{\circledR}-24$ Sample Preparation System, MP Biomedicals, Santa Ana, CA, USA) at the setting of $6 \mathrm{~m} \cdot \mathrm{s}^{-1}$ and duration of $1 \mathrm{~min}$ until all cells were found broken under the microscope (cycle number $=3)$. The homogenate was centrifuged at $12,000 \mathrm{~g} 4^{\circ} \mathrm{C}$ for $10 \mathrm{~min}$ to obtain the supernatant. The activities of total superoxide dismutase (SOD), catalase (CAT), and nitric oxide synthetase (NOS) in the supernatants were measured using commercial kits (A001, A007, and A014, Nanjing Jiancheng Bioengineering Institute, Nanjing, China), following the manufacturer's recommendations. Then, the concentration of total protein in the supernatant was quantified with
BCA Protein Assay kit (Sangon Biotech, Shanghai, China) and used to normalize the measured activities of SOD, CAT, and NOS to $\mathrm{U} \cdot \mathrm{mg}^{-1}$ protein.

The caspase 3 activity in the supernatant was measured by caspase3 Colorimetric Assay Kit (KeyGen Biotech, Nanjing, China) according to the manufacturer's protocol. Briefly, $50 \mu \mathrm{L}$ reaction buffer and $5 \mu \mathrm{L}$ substrate were added in $50 \mu \mathrm{L}$ supernatant, followed by incubation in the dark at $37^{\circ} \mathrm{C}$ for $4 \mathrm{~h}$. Then, the color change was measured spectrophotometrically at a wavelength of $405 \mathrm{~nm}$. The activity of caspase 3 was defined as the absorbance of the reaction solution at $405 \mathrm{~nm}$ (A405), and its activation level of caspase 3 in algal cells was defined as the ratio of A405 in samples to that of the $\mathrm{N}$ - group.

Measurement of cellular carbon and nitrogen. GF/F glass microfiber filters wrapped with aluminum foil were pre-combusted at $450^{\circ} \mathrm{C}$ for $3-5 \mathrm{~h}$ in a Muffle Furnace. Samples were collected at $84 \mathrm{~h}$ after the treatments, and a $7-\mathrm{mL}$ sample was filtered onto the pre-combusted filter. The total mass of carbon or nitrogen in the filter was measured using PE2400 SERIESII CHNS/O Elemental Analyzer and calculated as per cell content.

$R N A$ extraction and transcriptome sequencing (RNA-seq). At the end of the experimental treatments (cultured for $84 \mathrm{~h}$ ), $10^{7}$ cells from each culture were harvested by centrifugation at $5000 \mathrm{~g} 4^{\circ} \mathrm{C}$ for $10 \mathrm{~min}$ and resuspended in $1 \mathrm{~mL}$ TRIReagent (Molecular Research Center, Inc., Cincinnati, Ohio, USA). RNA extraction was carried out using TRI-Reagent combined with the Qiagen RNeasy Mini kit (Qiagen, Valencia, CA, USA) following a previously reported protocol (Lin et al. 2010). RNA concentrations of the samples were measured using NanoDrop ND-2000 spectrophotometer (Thermo Scientific, Wilmington, DE, USA), and the RNA quality was evaluated using the RNA 6000 Nano LabChip Kit in microcapillary electrophoresis (Agilent 2100 Bioanalyzer, Agilent Technologies, Santa Clara, CA, USA).

Twelve paired-end fragment libraries $(2 \times 100 \mathrm{bp}$, three replicates each group) were constructed and sequenced on the BGISEQ-500 platform according to the manufacturer's instructions (BGI, Shenzhen, China). The generated raw sequencing reads have been deposited at the NCBI Short Read Archive (SRA) BioProject, under accession: PRJNA510645.

Identification and annotation of differentially expressed genes. Raw reads were first processed using the Fastx-toolkit pipeline (http://hannonlab.cshl.edu/fastx_toolkit/index.html) to summarize data production, evaluate sequencing quality, and remove low-quality reads and adaptor sequences. Quality filtration removed sequences in which $80 \%$ of the base pairs had a Phred score of $<20$, while adaptor sequences were removed using the trimmer package. The genome sequences, genome annotation, and protein sequences of Cladocopium goreaui available at a repository for marine genomics data (http://symbs. reefgenomics.org/; Liu et al. 2018) were used as the reference database. The alignment of paired-end reads to the reference was performed using the HISAT2.2.1 software with default parameters (Pertea et al. 2016). StringTie v2.0 and DESeq2 software were used to estimate transcript abundances and identify differentially expressed genes (DEGs) for the comparisons of $\mathrm{N}+/ \mathrm{N}-$, Heat_N-/N-, Heat_N+/N-, and Heat_N $+/$ Heat_N(FDR < 0.05; Love et al. 2014).

After the identification of DEGs, their protein sequences were retrieved to align to the non-redundant protein database $(\mathrm{Nr})$ using the online BLASTP program (https://blast. ncbi.nlm.nih.gov/Blast.cgi). Furthermore, these proteins' domains were further predicted through Simple Modular Architecture Research Tool 8.0 (SMART, http://smart.emblheidelberg.de/; Schultz et al. 1998). These protein homologues and possible domains were both used for functional annotation of our DEGs. 
Gene Ontology (GO) overrepresentation and network analysis of DEGs. For GO analysis, sequences of all 35,913 Symbiodiniaceae proteins were aligned by local BLASTP search to the UniProt database with the threshold evalue $<0.001$ and analyzed using InterProScan software to predict their domains (UniProt 2015). The results obtained were parsed using Blast2GO software (https://www.blast2go.com/) for assigning GO terms. GO overrepresentation analysis was implemented via the hypergeometric test with a filter value of $0.05(\mathrm{~N}+/ \mathrm{N}-$ and Heat_N-/N- comparisons) or 0.001 (Heat_N+/ Heat_N- comparison). The significantly up-regulated or down-regulated genes were selected as the test set, while all genes were used as the reference set. The BiNGO tool was employed to calculate the overrepresented GO terms in the network and display them as a network of significant GO terms (Maere et al. 2005). Based on the mRNA expression level (FPKM, Fragments Per Kilobase of exon per Million fragments mapped) of DEGs in all transcriptome libraries, the WGCNA package was employed to construct a weighted correlation network (Langfelder and Horvath 2008). The network was further analyzed and displayed in the Cytoscape software (Shannon et al. 2003).

Statistical analysis. All data were presented as means $\pm \mathrm{s}$ tandard deviation (SD). The data of cell concentration, cell size, and enzyme activities during the whole experiment process were subjected to a two-way analysis of variance (two-way ANOVA) followed by multiple comparisons (S-N-K) using SPSS v22.0 to determine the significant effects of different treatments and times. For cell concentration, chlorophyll $a$ content, photochemical efficiency, and cellular nitrogen and carbon content at $84 \mathrm{~h}$, the data were subjected to one-way ANOVA and S-N-K multiple comparisons to determine significant differences among different treatments. Differences were considered significant at $P<0.05$.

\section{RESULTS}

Temporal trends of algal concentration and cell size under nitrogen supplement and high temperature. Nitrogen supplement increased the population growth of Cladocopium goreaui, whether under elevated or normal temperature (Fig. 1A). The highest level of cell concentration occurred in the $\mathrm{N}+$ group $\left(11.01 \pm 1.24 \times 10^{4}\right.$ cells $\cdot \mathrm{mL}^{-1}$; ANOVA, $F_{3,15}=$ $67.365, P<0.05)$ at $84 \mathrm{~h}$ after the treatments. The cell concentration in the Heat_N+ group was $8.28 \pm 0.63 \times 10^{4}$ cells $\cdot \mathrm{mL}^{-1}$, which was also significantly higher than those in the $\mathrm{N}-(4.91 \pm$ $0.45 \times 10^{4}$ cells $\left.\cdot \mathrm{mL}^{-1}\right)$ and Heat_N $-(5.10 \pm 0.54$ $\times 10^{4}$ cells $\cdot \mathrm{mL}^{-1}$ ) groups (Fig. $1 \mathrm{~B}$ ). However, no significant differences were observed in the cell size under the different conditions used during the whole treatment process (Fig. 1C).

Effects of nitrogen supplement and high temperature on photosynthesis parameters. At $84 \mathrm{~h}$ after the treatments, the highest level of photochemical efficiency $\mathrm{Fv} / \mathrm{Fm}$ was observed in the Heat_N- group $(0.90 \pm$ 0.03 ; ANOVA, $\left.F_{3,14}=27.566, P<0.05\right)$, and it was significantly higher than those that in the other three groups (Fig. 1D). However, chlorophyll $a$ content in this group $\left(0.12 \pm 0.01 \mathrm{pg} \cdot\right.$ cell $^{-1}$; ANOVA, $\left.F_{3,14}=10.762, P=0.01\right)$ was significantly lower than that in the $\mathrm{N}+$ group $\left(0.17 \pm 0.03 \mathrm{pg} \cdot \mathrm{cell}^{-1}\right)$ and the Heat_N+ group $\left(0.16 \pm 0.03 \mathrm{pg} \cdot \mathrm{cell}^{-1}\right)$ group.
There was no significant difference in chlorophyll $a$ content between the Heat_N- and $\mathrm{N}-$ groups (Fig. 1E).

Antioxidative enzyme activities under nitrogen supplement and high temperature. No significant difference was observed in SOD activity when the $\mathrm{N}+$, Heat_N-, and Heat_N+ groups were compared with the $\mathrm{N}$ - group (Fig. 2A). However, the CAT activity was significantly higher in the Heat_N- group $\left(67.98 \pm 16.72 \mathrm{U} \cdot \mathrm{mg}^{-1}\right)$ than the Heat_N+ group $\left(47.29 \pm 7.90 \mathrm{U} \cdot \mathrm{mg}^{-1}\right)$ at $24 \mathrm{~h}$, but not in other conditions (Fig. 2B). The NOS activity in the $\mathrm{N}+$ group was markedly lower than that in the $\mathrm{N}$ - group (control group) in the first $3 \mathrm{~d}$, and the difference diminished at $84 \mathrm{~h}$ (Fig. 2C). Similarly, the NOS activity in the Heat_N+ group was notably lower than in the $\mathrm{N}-$ group in the first 3 days. However, there was no significant difference in the NOS activity between the Heat_N + and Heat_N- groups during the whole experiment except at $24 \mathrm{~h}$, when the activity in the Heat_N+ group $\left(4.64 \pm 0.89 \mathrm{U} \cdot \mathrm{mg}^{-1}\right)$ was significantly lower than that in the $\mathrm{N}-$ group (Fig. 2C). Overall, the effects of the treatments on NOS activity disappeared at $84 \mathrm{~h}$.

Caspase 3 activation level under nitrogen supplement and high temperature. The effects of nitrogen supplement and temperature increase on caspase 3 activation were observed mostly on and after $72 \mathrm{~h}$ of treatment (Fig. 2D). The activation level in both the $\mathrm{N}+$ and Heat_N+ groups decreased significantly during the treatment period of 72-84 h, compared to those in the $\mathrm{N}-$ group (control group). The greatest decrease was observed at $72 \mathrm{~h}$ in the $\mathrm{N}+$ group $(0.56 \pm 0.19$-fold $)$ and $84 \mathrm{~h}$ in the Heat_ $\mathrm{N}+$ group $(0.43 \pm 0.07$-fold $)$. However, the caspase 3 activation level in the Heat_N- group (2.38 \pm 0.56 -fold) was significantly higher than that in the $\mathrm{N}-$ group only at $84 \mathrm{~h}$.

Effects of nitrogen supplement and high temperature on cellular nitrogen and carbon contents. The only significant difference in nitrogen content was observed in the Heat_N + group, which was higher $\left(0.97 \pm 0.14 \mathrm{ng} \cdot\right.$ cell $^{-1} ;$ ANOVA, $F_{3,15}=8.202$, $P=0.02$ ) than that in the $\mathrm{N}-$ group (control group, $0.81 \pm 0.05 \mathrm{ng} \cdot$ cell $^{-1}$ ) at $84 \mathrm{~h}$ (Fig. 3A). Nitrogen contents in the $\mathrm{N}+$, Heat_N-, and $\mathrm{N}-$ groups were all similar. In contrast, the carbon contents in the $\mathrm{N}+\left(4.66 \pm 0.32 \mathrm{ng} \cdot \mathrm{cell}^{-1}\right)$ and Heat_N+ groups $\left(6.26 \pm 0.90 \mathrm{ng} \cdot \mathrm{cell}^{-1}\right)$ were both significantly lower than that in the $\mathrm{N}-$ group $\left(9.43 \pm 0.53 \mathrm{ng} \cdot \mathrm{cell}^{-1} ;\right.$ ANOVA, $F_{3,15}=62.737$, $P<0.05)$. Furthermore, while carbon contents in the N- and the Heat_N- groups were similar, the content in the Heat_N+ group was higher than that in the $\mathrm{N}+$ group (Fig. 3B).

Gene expression of algal cells under nitrogen supplement and high temperature. Twelve paired-end $(2 \times$ $100 \mathrm{bp})$ transcriptome libraries were sequenced, with three replicates for each group $(\mathrm{N}-, \mathrm{N}+$, Heat_N-, and Heat_N+ groups). All libraries were 
A

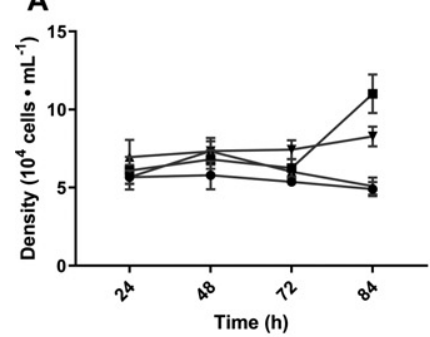

D

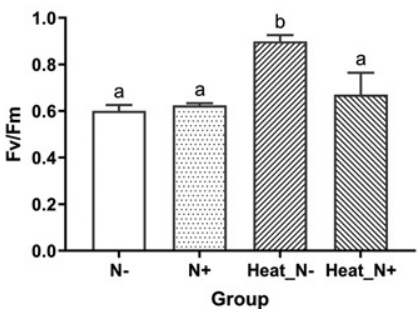

B
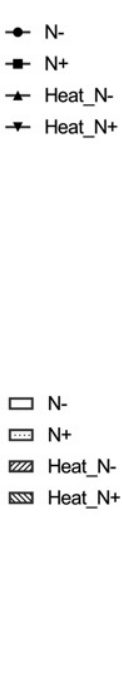
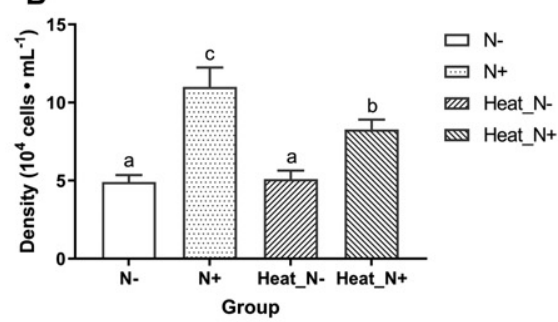

C

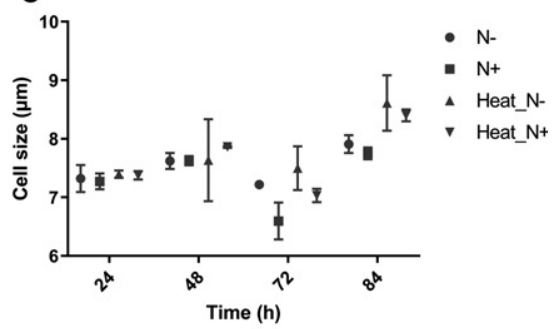

FIG. 1. Variations of cell physiology of cultured Cladocopium goreaui under nitrogen deficiency and high temperature. (A) Heat map of algal cell density at different times after culturing. (B) Histogram of cell density at $84 \mathrm{~h}$ after culturing. (C) Graph of algal cell size at different times after culturing. (D) Photochemical efficiency Fv/Fm at $84 \mathrm{~h}$ after culturing. (E) Chlorophyll $a$ content at $84 \mathrm{~h}$ after culturing. The $\mathrm{N}-, \mathrm{N}+$, Heat_N-, and Heat_N+ groups represent C. goreaui under nitrogen deficiency, nitrogen supplement, nitrogen deficiency with high temperature, and nitrogen supplement with high temperature, respectively. Data points represent means and error bars represent standard deviations $(N=5)$. Bars with different letters were significantly different $(P<0.05)$.
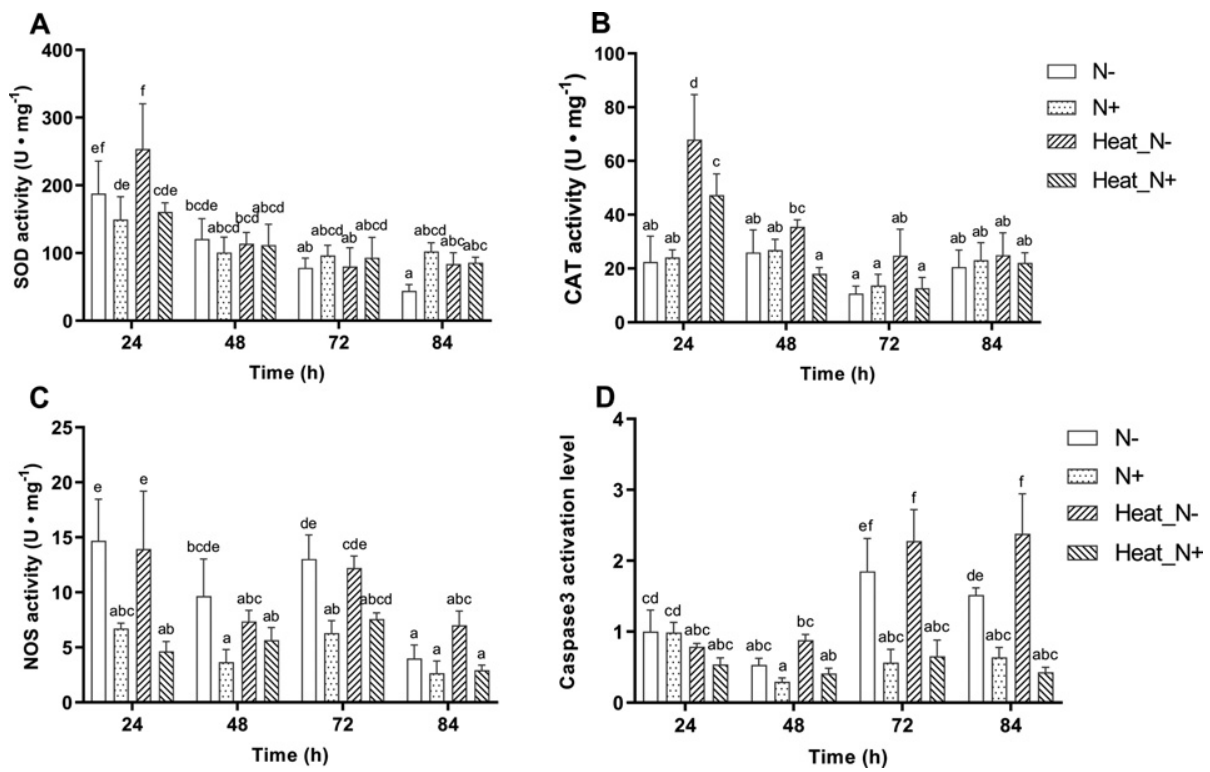

FIG. 2. Temporal patterns of superoxide dismutase (SOD, A), catalase (CAT, B), nitric oxide synthase (NOS, C) activities, and caspase3 activation level (D) in cultured Cladocopium goreaui under nitrogen deficiency and high temperature. The $\mathrm{N}-$, $\mathrm{N}+$, Heat_N-, and Heat_N+ groups represent C. goreaui under nitrogen deficiency, nitrogen supplement, nitrogen deficiency with high temperature, and nitrogen supplement with high temperature, respectively. Data points represent means, and error bars represent standard deviations $(N=5)$. Bars with different letters were significantly different $(P<0.05)$.

sequenced to saturation to reveal gene expression differences among nitrogen supply and high temperature conditions. After strict quality control, a total of 362,814,453 high-quality reads were obtained, about 25-35 million reads per library (Table 1). These clean reads were mapped to the reference genome of Cladocopium goreaui using HISAT2 software. Their mapping rates ranged from $87.55 \%$ to $89.07 \%$, yielding 22,265 to 22,638 genes (Table 1), indicating that most of the genome's encoding genes were represented in the transcriptomes. 

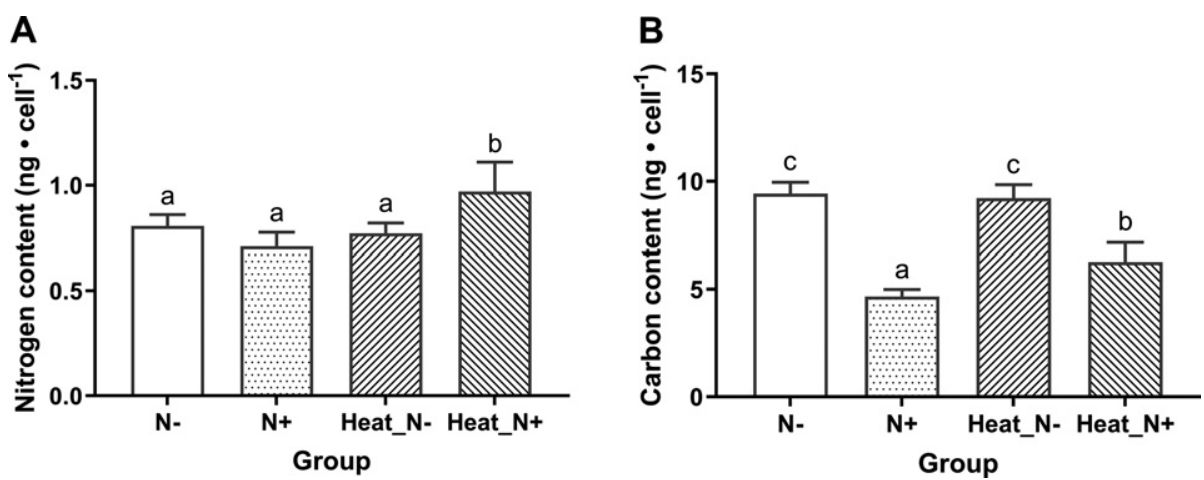

FIG. 3. Nitrogen (A) and carbon (B) content of cultured Cladocopium goreaui at $84 \mathrm{~h}$ under nitrogen deficiency and high temperature. The $\mathrm{N}-, \mathrm{N}+$, Heat_N-, and Heat_N+ groups represent C. goreaui under nitrogen deficiency, nitrogen supplement, nitrogen deficiency with high temperature, and nitrogen supplement with high temperature, respectively. Data points represent means, and error bars represent standard deviations $(N=5)$. Bars with different letters were significantly different $(P<0.05)$.

TABLE 1. Transcriptome mapping statistics.

\begin{tabular}{lcll}
\hline \hline Library & Total reads & Mapped rate & Detected genes \\
\hline N-_1 & $35,455,373$ & $89.07 \%$ & 22,270 \\
N-_2 & $35,487,428$ & $88.26 \%$ & 22,638 \\
$\mathrm{~N}-$-3 & $28,347,275$ & $87.83 \%$ & 22,571 \\
$\mathrm{~N}+$ 1 & $29,805,336$ & $88.63 \%$ & 22,265 \\
$\mathrm{~N}+$-2 & $32,258,651$ & $88.31 \%$ & 22,493 \\
$\mathrm{~N}+3$ & $24,770,369$ & $87.79 \%$ & 22,350 \\
Heat_N-_1 & $29,603,195$ & $87.55 \%$ & 22,563 \\
Heat_N-_2 & $27,899,840$ & $87.70 \%$ & 22,548 \\
Heat_N-_3 & $28,923,643$ & $87.73 \%$ & 22,527 \\
Heat_N+_1 & $30,663,799$ & $87.75 \%$ & 22,453 \\
Heat_N+_2 & $32,261,682$ & $87.90 \%$ & 22,477 \\
Heat_N+_3 & $27,337,862$ & $87.67 \%$ & 22,364 \\
\hline
\end{tabular}

The resulting mapped BAM files were fed into StringTie software to export the counts of mapped reads for each gene, which were further used by DESeq2 software to identify DEGs (Table S1 in the Supporting Information). All DEGs in the four comparisons of $\mathrm{N}+/ \mathrm{N}-$, Heat_N-/N-, Heat_N $+/ \mathrm{N}-$, and Heat_N+/Heat_N-were retrieved and analyzed (Figs. S1-S4 in the Supporting Information), and there were 301 (152 up-regulated, 149 down-regulated), 352 (235 up-regulated, 117 down-regulated), 1,397 (676 up-regulated, 721 down-regulated), and 4,371 (2,555 up-regulated, 1,816 down-regulated) DEGs in the four comparisons, respectively (Table 2, Tables S2-S5 in the Supporting Information).

Functional profile of differentially expressed genes under nitrogen supplement and high temperature. In the comparison of $\mathrm{N}+/ \mathrm{N}-$ libraries, a total of 301 DEGs were detected, comprised of 152 up-regulated and 149 down-regulated ones. The up-regulated DEGs did not overrepresent any GO terms, but the downregulated DEGs were enriched in eight GO terms, mainly involved in fatty acid biosynthesis mediated by acetyl-CoA carboxylase and ammonium transmembrane transport (Fig. 4A, Table S6 in the Supporting Information).
TABLE 2. The number of differentially expressed genes (DEGs) under nitrogen deficiency and high temperature.

\begin{tabular}{lcccc}
\hline \hline Comparisons & $\begin{array}{c}\mathrm{N}+/ \\
\mathrm{N}-\end{array}$ & $\begin{array}{c}\text { Heat_N-/ } \\
\mathrm{N}-\end{array}$ & $\begin{array}{c}\text { Heat_N+/ } \\
\mathrm{N}-\end{array}$ & $\begin{array}{c}\text { Heat_N+/ } \\
\text { Heat_N- }\end{array}$ \\
\hline Total DEGs & 301 & 352 & 1,397 & 4,371 \\
Up-regulated & 152 & 235 & 676 & 2,555 \\
$\quad \begin{array}{l}\text { DEGs } \\
\text { Down-regulated } \\
\text { DEGs }\end{array}$ & 149 & 117 & 721 & 1,816 \\
\hline
\end{tabular}

The Heat_N-/N- comparison revealed 235 upregulated and 117 down-regulated DEGs. The upregulated DEGs were overrepresented in $4 \mathrm{GO}$ terms related to AP-type membrane coat adaptor complex and motor activity (Table S7 in the Supporting Information). The down-regulated DEGs, in contrast, were mainly overrepresented in 11 GO terms that are primarily related to substrate-specific transmembrane transport and response to an organic substance (Fig. 4B, Table S8 in the Supporting Information).

Many more DEGs were found, 2,555 up-regulated and 1,816 down-regulated, in Heat_N+/Heat_Ncomparison, indicating the nutrient and heat interactive effects. Twenty-nine GO terms overrepresented by the up-regulated DEGs function in ion transport, photosynthetic endomembrane, and basal metabolism (Fig. 4C, Table S9 in the Supporting Information). Furthermore, $11 \mathrm{GO}$ terms related to protein binding and microtubule motor activity were found in the down-regulated DEGs in the Heat_N+/Heat_N- comparison (Table S10 in the Supporting Information). Besides, malate dehydrogenase, the enzyme known to be important in heat resistance in invertebrates (Dong et al. 2018) and plants (Kaur and Nainawatee 1989), was significantly up-regulated in the Heat_N+ condition relative to the Heat_N- condition. This result suggested that nitrogen nutrient deficiency might reduce the capacity to resist heat stress. 

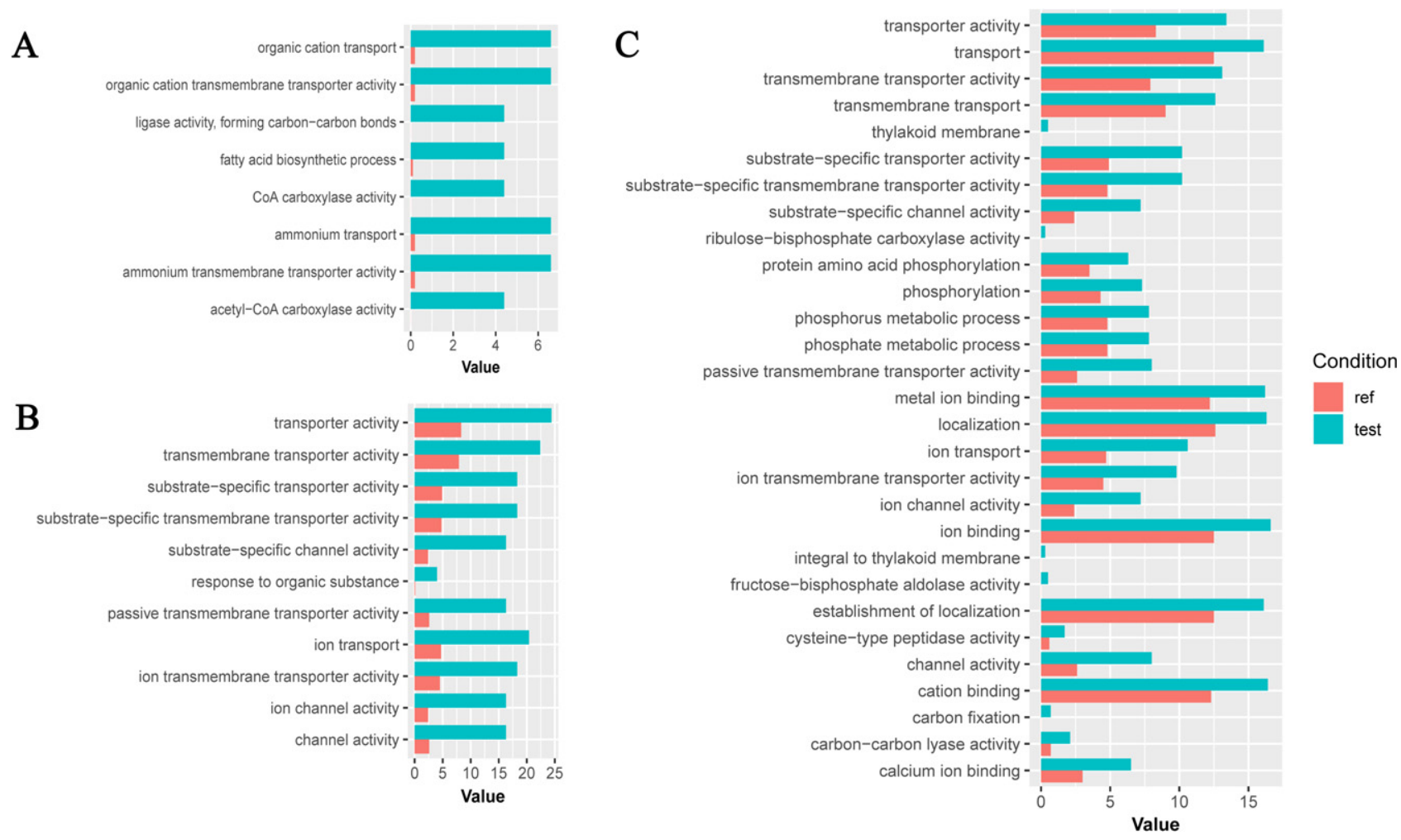

FIG. 4. Overrepresented GO terms of the differentially expressed genes of cultured Cladocopium goreaui at $84 \mathrm{~h}$ under nitrogen deficiency and high temperature. The $\mathrm{N}-, \mathrm{N}+$, Heat_N-, and Heat_N+ groups represent C. goreaui under nitrogen deficiency, nitrogen supplement, nitrogen deficiency with high temperature, and nitrogen supplement with high temperature, respectively. (A) the downregulated DEGs in the $\mathrm{N}+/ \mathrm{N}-$ comparison; (B) the down-regulated DEGs in the Heat_N-/N- comparison; and (C) the up-regulated DEGs in the Heat_N+/Heat_N- comparison. "Ref" refers to the proportion of genes functionally assigned a GO term out of all genes existing in the reference genome, while "test" refers to the proportion of genes assigned to that GO term out of the differentially expressed genes. [Color figure can be viewed at wileyonlinelibrary.com]

Network analysis of DEGs under nitrogen supplement and high temperature. We merged all DEGs in the $\mathrm{N}+/ \mathrm{N}-$, Heat_N-/N-, and Heat_N $+/ \mathrm{N}-$ comparisons, removed redundancy, and obtained 1,709 unique DEGs. Based on their expression level in all transcriptome libraries, a weighted gene co-expression network was constructed with five modules (Figs. 5A and $\mathrm{S} 5$ in the Supporting Information). Weight threshold $=0.35$ was used to refine the network, and a subnetwork was retrieved, which was comprised of 64 nodes (genes) and 167 edges. Venn analysis revealed 20 and 23 genes in the refined subnetwork that were exclusively DEGs in the Heat_N-/N- and Heat_N $+/ \mathrm{N}-$ comparisons, respectively. In addition, there were 21 common genes in the subnetwork and between the above two comparisons (Fig. 5B, Table S11 in the Supporting Information).

After removing genes (nodes) and edges with no functional annotation, the subnetwork was displayed using the Cytoscape software (Fig. 5C). We observed three genes encoding signal pathway-related proteins, including GTP-binding nuclear protein and two AP-1 complex subunit gamma. We also found three crucial hub genes, which encoded Copia protein (SymbC1.scaffold401.1, 38 edges), NippedB-like protein (SymbC1.scaffold509.2, 23 edges) a nd 1-acyl-sn-glycerol-3-phosphate acyltransferase (SymbC1.scaffold1982.5, 23 edges). According to functional annotation, the rest of the genes fell into three categories: synthesis and modification of nucleic acids, metabolism enzyme, and other functions. Notably, there were three DNA methyltransferase and two transporter (for calcium and UDPgalactose/glucose) genes in the subnetwork.

\section{DISCUSSION}

There is evidence that scleractinian coral can control the growth of symbiotic Symbiodiniaceae and promote its photosynthetic organic carbon output by regulating nitrogen supply (Radecker et al. 2015). In the present study, we found that nitrogen limitation promoted apoptosis in Cladocopium goreaui to suppress its growth and enhanced its carbon assimilation. High temperature stress imposed upon nitrogen limitation further induced antioxidant ability and apoptosis of C. goreaui, whereas nitrogen supplement reduced the negative effect of high temperature stress on algal cells through repressed 
A

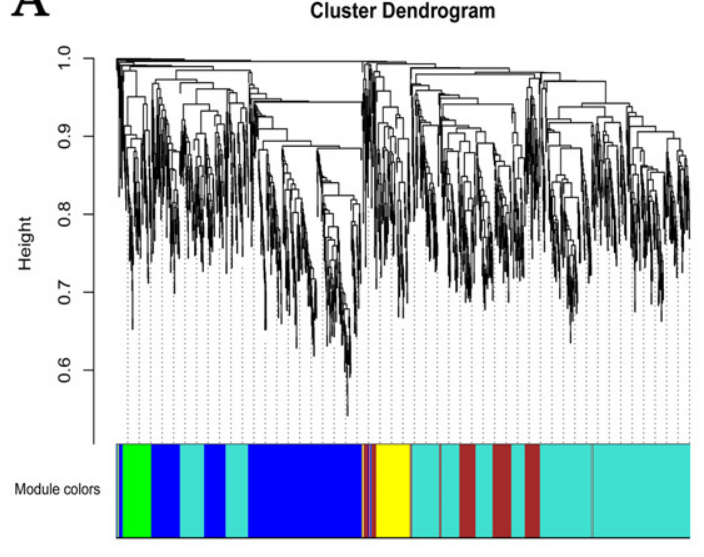

B

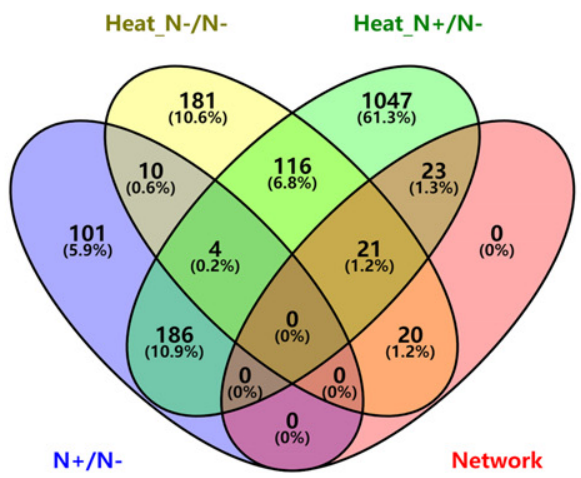

C

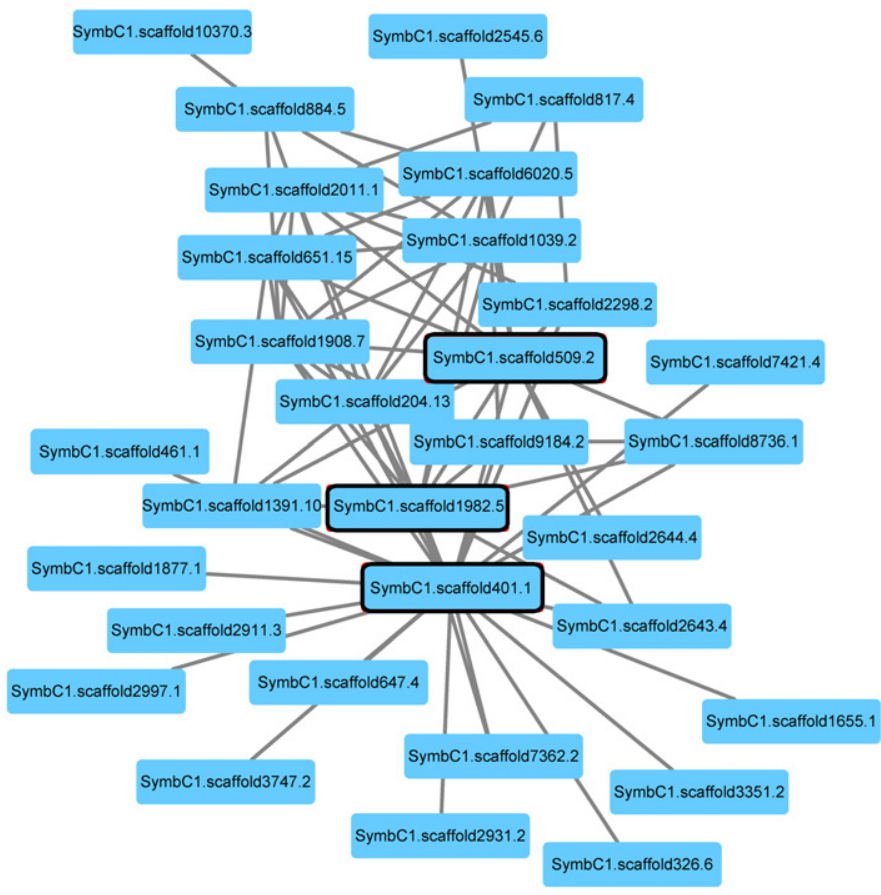

Fig. 5. (A) The cluster dendrogram of all the differentially expressed genes in the $\mathrm{N}+/ \mathrm{N}-$, Heat_N-/ N-, and Heat-N $+/ \mathrm{N}-$ comparisons, based their expression levels in twelve transcriptome libraries. (B) grouping of the differentially expressed genes in the $\mathrm{N}+/ \mathrm{N}-$, Heat_N-/N-, Heat-N $+/ \mathrm{N}-$ comparisons and weighted gene co-expression network. (C) Weighted gene co-expression network (threshold $=0.35)$ of all differentially expressed genes on their expression level in all transcriptome libraries and exhibited in the Cytoscape software. The boxes with bold border label three crucial hub genes. Three crucial hub genes included Copia protein (SymbC1.scaffold401.1, 38 edges), Nipped-B-like protein (SymbC1.scaffold509.2, 23 edges), and 1-acyl-sn-glycerol-3-phosphate acyltransferase (SymbC1.scaffold1982.5, 23 edges). [Color figure can be viewed at wileyonlinelibrary.com]

apoptosis, promoted ion transport, and optimized metabolism.

Suppressed growth, promoted carbon assimilation and apoptosis of Cladocopium goreaui under nitrogen limitation. The comparison between the $\mathrm{N}+$ and $\mathrm{N}-$ groups was employed to reveal the effect of nitrogen limitation on the physiological activities of Cladocopium goreaui. Compared to the $\mathrm{N}+$ group, we observed a significant decrease in cell concentration and chlorophyll $a$ content, and a significant increase in NOS activity, caspase3 activation level, and cellular carbon content in the $\mathrm{N}-$ group. These results demonstrated that nitrogen limitation repressed the growth and chlorophyll $a$ content in cultured C. goreaui and meanwhile induced NO production, apoptosis, and the accumulation of photosynthetically assimilated carbon. Symbiodiniaceae yield reduction under nitrogen limitation has previously been reported (Jiang et al. 2014), and this could be due to the elevation of NO production and apoptosis observed in the present study, as NO has been implicated in the heat stress-induced cell death of Symbiodinium microadriaticum (Bouchard and
Yamasaki 2009). The continuous photosynthetic carbon assimilation under nitrogen limitation, as previously reported in dinoflagellates (Jing et al. 2017), is consistent with the observations of lipid droplet accumulation in Symbiodiniaceae by nitrogen deprivation (Jiang et al. 2014, Weng et al. 2014) and the enrichment of GO terms related to fatty acid biosynthesis by the significantly down-regulated genes in the $\mathrm{N}+/ \mathrm{N}-$ comparison. Furthermore, our GO enrichment analysis showed that the expression levels of three ammonium transporter genes in C. goreaui increased under nitrogen limitation. This is, interestingly, although the $\mathrm{N}+$ cultures were provided nitrate instead of ammonium, but consistent with the fact that Symbiodiniaceae possess more ammonium transporter genes than nitrate transporter genes (Lin et al. 2015) and the proposition that ammonium is the main nitrogen resource for Symbiodiniaceae (Radecker et al. 2015). Taken together, the results from the physiological, biochemical, and gene expression measurements suggest that nitrogen limitation can restrict the growth of cultured C. goreaui probably through NO 
production and apoptosis induction, but allow continued photosynthetic carbon assimilation and accumulation in the Symbiodiniaceae cells.

Heat induction of antioxidant and apoptosis level of Cladocopium goreaui under nitrogen limitation. To understand how Cladocopium goreaui responds to high temperature under nitrogen limitation, we compared various physiological parameters between the Heat_N- and $\mathrm{N}-$ groups. Fv/Fm, CAT activity, and caspase 3 activation levels in the Heat_N- group were significantly higher than those in the $\mathrm{N}-$ group at 84,24 , and $84 \mathrm{~h}$ after culturing, respectively. The rise of Fv/Fm under Heat_N- was a surprise, and the underlying mechanism is unclear, but this, along with the unchanged carbon content in C. goreaui, indicated that photosynthesis was not significantly damaged by the heat and N-deficiency combined treatment. The increase in CAT activity could have resulted from increased production of $\mathrm{H}_{2} \mathrm{O}_{2}$ in cultured $C$. goreaui under Heat_N-, as heat stress has been reported to induce $\mathrm{H}_{2} \mathrm{O}_{2}$ production in a symbiotic dinoflagellate Symbiodinium spp (Suggett et al. 2008). If $\mathrm{H}_{2} \mathrm{O}_{2}$ reacted with $\mathrm{NO}$, it could promote apoptosis of $C$. goreaui.

DEGs analysis in the comparison of Heat_N-/ $\mathrm{N}-$ could provide insights about whether nitrogen limitation aggravates heat-induced caspase3 activation of cultured Cladocopium goreaui. Genes associated with clathrin-coated vesicles were significantly up-regulated under Heat_N-, suggesting induction of phagocytosis in C. goreaui because clathrin-coated vesicles typically function in endocytosis (Ehrlich et al. 2004). The overrepresented GO terms for the significantly down-regulated genes were mainly related to transmembrane transport of ions, including chloride, magnesium, potassium, sodium, and calcium. The down-regulated expression of these ion channel genes could contribute to the apoptosis induction of cultured $C$. goreaui because transmembrane ion flux has been reported to be associated with the change of cell state and cell cycle in dinoflagellates (Gordeeva et al. 2004, Yeung et al. 2006, Smith et al. 2011). Therefore, although no significant change in the cell yield was observed during our experimental period, the apoptosis of algal cells could be induced and would eventually result in the decrease of cell yield, as commonly observed in the symbiotic Symbiodiniaceae of scleractinian coral under heat stress (Strychar et al. 2004). Together, these results suggest that under nitrogen limitation and high temperature can activate stress response and induce antioxidant capacity and apoptosis level of $C$. goreaui to decrease its population, without apparent effect on photosynthesis.

Nitrogen availability lessened heat stress effects on Cladocopium goreaui through repressed apoptosis, transcriptional regulation, and optimized metabolism. Nitrogen nutrient elevation has been shown to alter coral-Symbiodiniaceae symbiosis's susceptibility to heat bleaching (D'Angelo and Wiedenmann 2014,
Fernandes de Barros Marangoni et al. 2020). However, little is known about the potential effect and mechanism of nitrogen availability on heat resistance or acclimation of symbiotic Symbiodiniaceae. To explore this issue, a comparison of the Heat_N+ group with the Heat_N- group is potentially insightful. In the present study, growth, chlorophyll $a$, and nitrogen content in the Heat_N+ group increased significantly at $24 \mathrm{~h}, 48 \mathrm{~h}$, and $84 \mathrm{~h}$ respectively, whereas NOS activity ( $24 \mathrm{~h})$, SOD activity $(24 \mathrm{~h})$, CAT activity $(24-48 \mathrm{~h})$, carbon content $(84 \mathrm{~h})$, and caspase3 activation level (72-84 h) decreased significantly in a temporarily sequential manner. These are evidence of reducing heat-induced stress by the availability of nitrogen.

The nitrogen content of Cladocopium goreaui cells in the Heat_N+ group was higher than all other treatment groups, indicating that nitrogen supplement can meet the increased demand for nitrogen to cope with heat stress. Furthermore, the increases in chlorophyll $a$ content and total carbon content, together with the enrichment of GO terms related to thylakoid membrane by the up-regulated DEGs in the Heat_N+/Heat_N- comparison, testify the enhancement of the photosynthesis of $C$. goreaui under nitrogen supplement. The supply of nitrogen and fixed carbon could support population growth in C. goreaui after heat stress. Furthermore, the repression of apoptosis owing to the dramatic decline of caspase 3 activation demonstrates that nitrogen availability repressed apoptosis promoted by nitrogen limitation and heat stress. The decrease of oxidative stress owing to the significant decreases of SOD, CAT, and NOS activities could also result in the repression of apoptosis of $C$. goreaui under this condition. Furthermore, other GO terms overrepresented by the up-regulated DEGs in the Heat_N+/Heat_Ncomparison were mainly related to ion transport, which were down-regulated in the Heat_N-/Ncomparison, demonstrating that nitrogen availability might promote ion transport and further repress apoptosis in C. goreaui. The enhancement of nitrogen and carbon assimilation, the promotion of ion transport, and the repression of apoptosis together contributed to the growth of $C$. goreaui under heat stress and nitrogen availability. Finally, the up-regulation of malate dehydrogenase under Heat_N+ also suggests enhanced heat resistance conferred by nitrogen nutrient supply. It has been reported that the structural stability and enzymatic activity of malate dehydrogenase are important for the thermal resistance in marine molluscs (Dong et al. 2018) and plants (Kaur and Nainawatee 1989). Therefore, nitrogen availability could reduce the negative effects of heat stress on symbiotic Symbiodiniaceae and may improve the resistibility of coral-Symbiodiniaceae symbiotic association to heat bleaching.

To further illuminate the core molecular mechanism underlying the role of nitrogen availability in acquiring heat resistance of symbiotic 


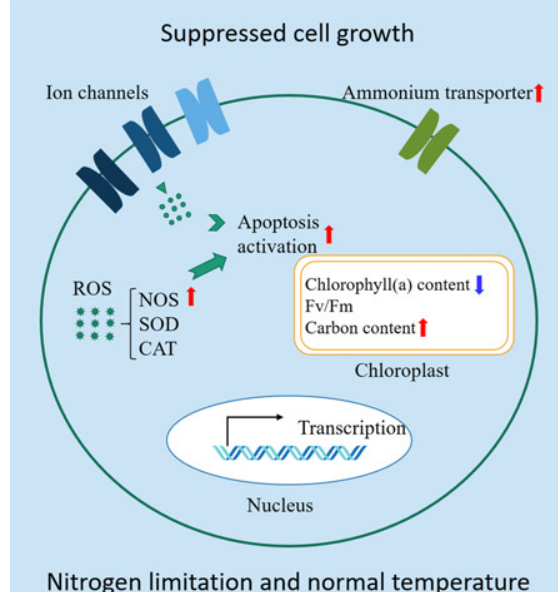

Nitrogen limitation and normal temperature $\mathrm{N}-/ \mathrm{N}+$
Promoted apoptosis

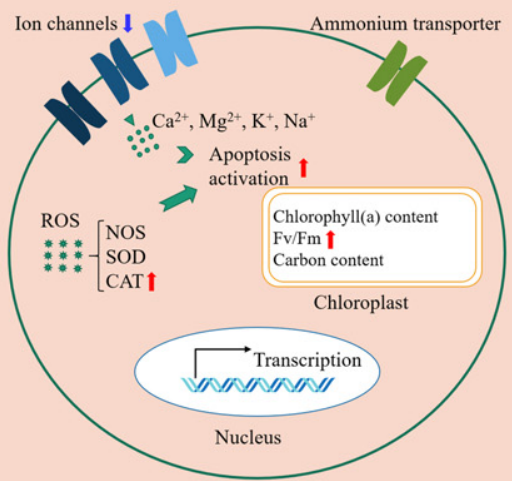

Nitrogen limitation and high temperature Heat_N-/N-
Suppressed apoptosis and promoted cell growth

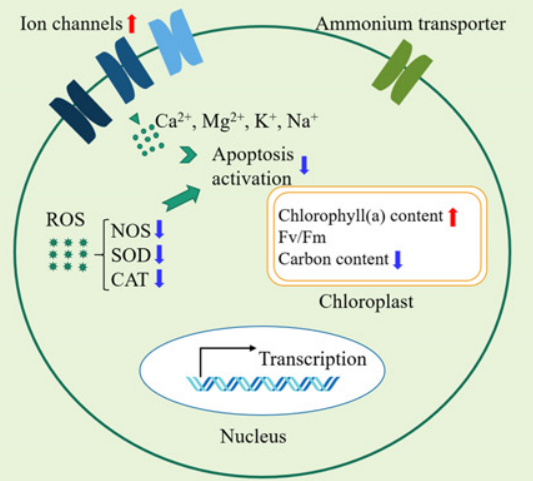

Nitrogen availability and High temperature Heat_N+/Heat_N-

FIg. 6. The physiological response patterns of symbiotic Symbiodiniaceae Cladocopium goreaui to high temperature under nitrogen limitation and availability. [Color figure can be viewed at wileyonlinelibrary.com]

Symbiodiniaceae, a weighted gene co-expression network of all differentially expressed genes was constructed and condensed (weight threshold $=0.35$ ). There were 64 nodes (genes) and 167 edges (reciprocities) in the core network. The network analysis also revealed that three crucial hub genes (most reciprocities), copia protein, Nipped-Blike protein, and 1-acyl-sn-glycerol-3-phosphate acyltransferase, were up-regulated in both Heat_N-/ $\mathrm{N}-$ and Heat_N+/N- comparisons, and all responsive to high temperature. Copia protein has been postulated to be heat-activated retrotransposons in S. microadriaticum (Chen et al. 2018). Among other genes in the network, the expression levels of three signal pathway molecules, including one small $G$ protein and two AP-1 proteins, were all elevated in the Heat_N-/N- comparison, whereas only one AP-1 protein was up-regulated in the Heat_N $+/ \mathrm{N}-$ comparison. Because AP-1 is an important transcription factor mediating the regulation of apoptosis activation under internal and external environment changes (Shaulian and Karin 2002, Ameyar et al. 2003), nitrogen availability could attenuate the induction of heat stress through the small $G$ protein/AP-1 pathway by down-regulating expression of these genes, which would increase the resilience of C. goreaui to high temperature. Similarly, two transporter genes and metabolic enzymes in the core network further highlight the nitrogen regulation of metabolisms and apoptosis in Symbiodiniaceae C. goreaui in response to heat stress. In sum, results from the present study suggest that nitrogen availability has the potential to improve the resilience of symbiotic C. goreaui to high temperature stress through a multitude of mechanisms (Fig. 6).

The authors were grateful to all of the laboratory members for their continuous technical advice and helpful discussions. Z.Z. gratefully acknowledges the financial support from China Scholarship Council (201708460042) to enable his visit to the University of Connecticut. This research was supported by grants (ZDKJ2019011) from the Major Science and Technology Program of Hainan Province, National Key R\&D Program of China (2018YFC1406500), National Science Foundation of China (Grant 31772460 and 31661143029), and Natural Science Foundation of Hainan Province (Grant 2019RC067).

\section{AUTHOR CONTRIBUTION}

Z. Zhou: Conceptualization (equal); data curation (equal); investigation (equal); visualization (equal); writing - original draft (equal). K. Zhang: Investigation (equal). L. Wang: Project administration (equal); supervision (equal). Y. Su: Data curation (equal); investigation (equal). J. Wang: Investigation (equal); methodology (equal). T. Song: Investigation (equal). X. Yang: Investigation (equal); methodology (equal). J. Tang: Software (equal); validation (equal); visualization (equal). S. Lin: Conceptualization (equal); supervision (equal); validation (equal); writing - review and editing (equal).

Ameyar, M., Wisniewska, M. \& Weitzman, J. B. 2003. A role for AP-1 in apoptosis: the case for and against. Biochimie 85:74752.

Arar, E. J. \& Collins, G. B. 1997. Method 445.0: in Vitro Determination of Chlorophyll a and Pheophytin a in Marine and Freshwater Algae by Fluorescence. Revision 1.2. National Exposure Research Laboratory Office of Research and development, U.S. Environmental Protection Agency, Cincinnati, $\mathrm{OH}$.

Bouchard, J. N. \& Yamasaki, H. 2009. Implication of nitric oxide in the heat-stress-induced cell death of the symbiotic alga Symbiodinium microadriaticum. Mar. Biol. 156:2209-20.

Chen, J. E., Cui, G., Wang, X., Liew, Y. J. \& Aranda, M. 2018. Recent expansion of heat-activated retrotransposons in the coral symbiont Symbiodinium microadriaticum. ISME J. 12:63943.

D'Angelo, C. \& Wiedenmann, J. 2014. Impacts of nutrient enrichment on coral reefs: new perspectives and implications for 
coastal management and reef survival. Curr. Opin. Env. Sust. 7:82-93.

Dong, Y. W., Liao, M. L., Meng, X. L. \& Somero, G. N. 2018. Structural flexibility and protein adaptation to temperature: molecular dynamics analysis of malate dehydrogenases of marine molluscs. Proc. Natl. Acad. Sci. USA 115:1274-9.

Downs, C. A., Fauth, J. E., Halas, J. C., Dustan, P., Bemiss, J. \& Woodley, C. M. 2002. Oxidative stress and seasonal coral bleaching. Free Radic. Biol. Med. 33:533-43.

Ehrlich, M., Boll, W., Van Oijen, A., Hariharan, R., Chandran, K., Nibert, M. L. \& Kirchhausen, T. 2004. Endocytosis by random initiation and stabilization of clathrin-coated pits. Cell 118:591-605.

Falkowski, P. G., Dubinsky, Z., Muscatine, L. \& Porter, J. W. 1984. Light and the bioenergetics of a symbiotic coral. Bioscience 34:705-9.

Fernandes de Barros Marangoni, L., Marangoni, L., Ferrier-Pages, C., Rottier, C., Bianchini, A. \& Grover, R. 2020. Unravelling the different causes of nitrate and ammonium effects on coral bleaching. Sci. Rep. 10:11975.

Gonzalez-Pech, R. A., Bhattacharya, D., Ragan, M. A. \& Chan, C. X. 2019. Genome evolution of coral reef symbionts as intracellular residents. Trends Ecol. Evol. 34:799-806.

Gordeeva, A. V., Labas, Y. A. \& Zvyagilskaya, R. A. 2004. Apoptosis in unicellular organisms: mechanisms and evolution. Biochem. 69:1055-66.

Hall, E. R., Muller, E. M., Goulet, T., Bellworthy, J., Ritchie, K. B. \& Fine, M. 2018. Eutrophication may compromise the resilience of the Red Sea coral Stylophora pistillata to global change. Mar. Pollut. Bull. 131:701-11.

Houlbreque, F. \& Ferrier-Pages, C. 2009. Heterotrophy in tropical scleractinian corals. Biol. Rev. Camb. Philos. Soc. 84:1-17.

Jiang, P. L., Pasaribu, B. \& Chen, C. S. 2014. Nitrogen-deprivation elevates lipid levels in Symbiodinium spp. by lipid droplet accumulation: morphological and compositional analyses. PLOS ONE 9:e87416.

Jing, X., Lin, S., Zhang, H., Koerting, C. \& Yu, Z. 2017. Utilization of urea and expression profiles of related genes in the dinoflagellate Prorocentrum donghaiense. PLoS One 12: e0187837.

Kaur, J. \& Nainawatee, H. S. 1989. Malate dehydrogenase activity of leaves of a thermotolerant Triticum aestivum mutant. Biochem. Physiol. Pflanz. 185:277-80.

LaJeunesse, T. C., Parkinson, J. E., Gabrielson, P. W., Jeong, H. J., Reimer, J. D., Voolstra, C. R. \& Santos, S. R. 2018. Systematic revision of Symbiodiniaceae highlights the antiquity and diversity of coral endosymbionts. Curr. Biol. 28:2570-80.

Langfelder, P. \& Horvath, S. 2008. WGCNA: an R package for weighted correlation network analysis. BMC Bioinformatics 9:559.

Lesser, M. P. 1997. Oxidative stress causes coral bleaching during exposure to elevated temperatures. Coral Reefs 16:187-92.

Li, M., Shi, X., Guo, C. \& Lin, S. 2016. Phosphorus deficiency inhibits cell division but not growth in the dinoflagellate Amphidinium carterae. Front. Microbiol. 7:826.

Lin, S., Cheng, S., Song, B., Zhong, X., Lin, X., Li, W., Li, L. et al. 2015. The Symbiodinium kawagutii genome illuminates dinoflagellate gene expression and coral symbiosis. Science 350:691-4.

Lin, S., Zhang, H., Zhuang, Y., Tran, B. \& Gill, J. 2010. Spliced leader-based metatranscriptomic analyses lead to recognition of hidden genomic features in dinoflagellates. Proc. Natl. Acad. Sci. USA 107:20033-8.

Lin, X., Zhang, H., Huang, B. \& Lin, S. 2012. Alkaline phosphatase gene sequence characteristics and transcriptional regulation by phosphate limitation in Karenia brevis (Dinophyceae). Harmful Algae 17:14-24.

Lin, Z., Chen, M., Dong, X., Zheng, X., Huang, H., Xu, X. \& Chen, J. 2017. Transcriptome profiling of Galaxea fascicularis and its endosymbiont Symbiodinium reveals chronic eutrophication tolerance pathways and metabolic mutualism between partners. Sci. Rep. 7:42100.
Liu, H., Stephens, T. G., Gonzalez-Pech, R. A., Beltran, V. H., Lapeyre, B., Bongaerts, P., Cooke, I. et al. 2018. Symbiodinium genomes reveal adaptive evolution of functions related to coral-dinoflagellate symbiosis. Commun. Biol. 1:95.

Love, M. I., Huber, W. \& Anders, S. 2014. Moderated estimation of fold change and dispersion for RNA-seq data with DESeq2. Genome Biol. 15:550.

Maere, S., Heymans, K. \& Kuiper, M. 2005. BiNGO: a Cytoscape plugin to assess overrepresentation of gene ontology categories in biological networks. Bioinformatics 21:3448-9.

Morris, L. A., Voolstra, C. R., Quigley, K. M., Bourne, D. G. \& Bay, L. K. 2019. Nutrient availability and metabolism affect the stability of coral-symbiodiniaceae symbioses. Trends Microbiol. 27:678-89.

Peng, S. E., Chen, C. S., Song, Y. F., Huang, H. T., Jiang, P. L., Chen, W. N., Fang, L. S. \& Lee, Y. C. 2012. Assessment of metabolic modulation in free-living versus endosymbiotic Symbiodinium using synchrotron radiation-based infrared microspectroscopy. Biol. Lett. 8:434-7.

Pernice, M., Meibom, A., Van Den Heuvel, A., Kopp, C., DomartCoulon, I., Hoegh-Guldberg, O. \& Dove, S. 2012. A singlecell view of ammonium assimilation in coral-dinoflagellate symbiosis. ISME J. 6:1314-24.

Pertea, M., Kim, D., Pertea, G. M., Leek, J. T. \& Salzberg, S. L. 2016. Transcript-level expression analysis of RNA-seq experiments with HISAT, StringTie and Ballgown. Nat. Protoc. 11:1650-67.

Radecker, N., Pogoreutz, C., Voolstra, C. R., Wiedenmann, J. \& Wild, C. 2015. Nitrogen cycling in corals: the key to understanding holobiont functioning? Trends Microbiol. 23:490-7.

Rosenberg, E., Koren, O., Reshef, L., Efrony, R. \& Zilber-Rosenberg, I. 2007. The role of microorganisms in coral health, disease and evolution. Nat. Rev. Microbiol. 5:355-62.

Schultz, J., Milpetz, F., Bork, P. \& Ponting, C. P. 1998. SMART, a simple modular architecture research tool: identification of signaling domains. Proc. Natl. Acad. Sci. USA 95:5857-64.

Shannon, P., Markiel, A., Ozier, O., Baliga, N. S., Wang, J. T., Ramage, D., Amin, N., Schwikowski, B. \& Ideker, T. 2003. Cytoscape: a software environment for integrated models of biomolecular interaction networks. Genome Res. 13:2498-504.

Shaulian, E. \& Karin, M. 2002. AP-1 as a regulator of cell life and death. Nat. Cell Biol. 4:E131-E136.

Shinzato, C., Shoguchi, E., Kawashima, T., Hamada, M., Hisata, K., Tanaka, M., Fujie, M. et al. 2011. Using the Acropora digitifera genome to understand coral responses to environmental change. Nature 476:320-3.

Smith, S. M., Morgan, D., Musset, B., Cherny, V. V., Place, A. R., Hastings, J. W. \& Decoursey, T. E. 2011. Voltage-gated proton channel in a dinoflagellate. Proc. Natl. Acad. Sci. USA 108:18162-7.

Strychar, K. B., Coates, M., Sammarco, P. W. \& Piva, T. J. 2004. Bleaching as a pathogenic response in scleractinian corals, evidenced by high concentrations of apoptotic and necrotic zooxanthellae. J. Exp. Mar. Biol. Ecol. 304:99-121.

Su, Y., Zhang, K., Zhou, Z., Wang, J., Yang, X., Tang, J., Li, H. \& Lin, S. 2020. Microplastic exposure represses the growth of endosymbiotic dinoflagellate Cladocopium goreaui in culture through affecting its apoptosis and metabolism. Chemosphere 244:125485.

Suggett, D. J., Warner, M. E., Smith, D. J., Davey, P., Hennige, S. \& Baker, N. R. 2008. Photosynthesis and production of hydrogen peroxide by Symbiodinium (Pyrrhophyta) phylotypes with different thermal tolerances. J. Phycol. 44:948-56.

Tanaka, Y., Suzuki, A. \& Sakai, K. 2018. The stoichiometry of coral-dinoflagellate symbiosis: carbon and nitrogen cycles are balanced in the recycling and double translocation system. ISME J. 12:860-8.

UniProt, C. 2015. UniProt: a hub for protein information. Nucleic Acids Res. 43:D204-D212.

Vidal-Dupiol, J., Ladriere, O., Destoumieux-Garzon, D., Sautiere, P. E., Meistertzheim, A. L., Tambutte, E., Tambutte, S., Duval, D., Foure, L., Adjeroud, M. \& Mitta, G. 2011. Innate 
immune responses of a scleractinian coral to vibriosis. J. Biol. Chem. 286:22688-98.

Weng, L. C., Pasaribu, B., Lin, I. P., Tsai, C. H., Chen, C. S. \& Jiang, P. L. 2014. Nitrogen deprivation induces lipid droplet accumulation and alters fatty acid metabolism in symbiotic dinoflagellates isolated from Aiptasia pulchella. Sci. Rep. 4:5777.

Wiedenmann, J., D’Angelo, C., Smith, E. G., Hunt, A. N., Legiret, F. E., Postle, A. D. \& Achterberg, E. P. 2012. Nutrient enrichment can increase the susceptibility of reef corals to bleaching. Nat. Clim. Change 3:160-4.

Yeung, P. K., Lam, C. M., Ma, Z. Y., Wong, Y. H. \& Wong, J. T. 2006. Involvement of calcium mobilization from caffeine-sensitive stores in mechanically induced cell cycle arrest in the dinoflagellate Crypthecodinium cohnii. Cell Calcium 39:259-74.

Yuan, C., Zhou, Z., Zhang, Y., Chen, G., Yu, X., Ni, X., Tang, J. \& Huang, B. 2017. Effects of elevated ammonium on the transcriptome of the stony coral Pocillopora damicornis. Mar. Pollut. Bull. 114:46-52.

Zhou, Z., Zhang, G., Chen, G., Ni, X., Guo, L., Yu, X., Xiao, C., Xu, Y., Shi, X. \& Huang, B. 2017. Elevated ammonium reduces the negative effect of heat stress on the stony coral Pocillopora damicornis. Mar. Pollut. Bull. 118:319-27.

\section{Supporting Information}

Additional Supporting Information may be found in the online version of this article at the publisher's web site:

Figure S1. PCA analysis of six transcriptome libraries in two groups $(\mathrm{N}-$ and $\mathrm{N}+$ ) of symbiotic Symbiodiniaceae Cladocopium goreaui.

Figure S2. PCA analysis of six transcriptome libraries in two groups ( $\mathrm{N}-$ and $\mathrm{N}-$ _Heat $)$ of symbiotic Symbiodiniaceae Cladocopium goreaui.

Figure S3. PCA analysis of six transcriptome libraries in two groups ( $\mathrm{N}-$ and $\mathrm{N}+$ _Heat $)$ of symbiotic Symbiodiniaceae Cladocopium goreaui.

Figure S4. PCA analysis of six transcriptome libraries in two groups ( $\mathrm{N}$-_Heat and $\mathrm{N}+$ _Heat) of symbiotic Symbiodiniaceae Cladocopium goreaui.
Figure S5. The network heat map plot of all the differentially expressed genes in the $\mathrm{N}+/ \mathrm{N}-$, Heat_N- $/ \mathrm{N}-$ and Heat- $+/ \mathrm{N}-$ comparisons, based their expression levels in twelve transcriptome libraries.

Table S1. The mapped reads number of algal genes in the twelve libraries.

Table S2. Differentially expressed genes in the comparison of $\mathrm{N}+/ \mathrm{N}-$ groups.

Table S3. Differentially expressed genes in the comparison of Heat_N-/N- groups.

Table S4. Differentially expressed genes in the comparison of Heat_N+/N- groups.

Table S5. Differentially expressed genes in the comparison of Heat_N+/Heat_N-groups.

Table S6. Overrepresented GO terms of significantly downregulated genes in the comparison of $\mathrm{N}+/ \mathrm{N}-$ groups.

Table S7. Overrepresented GO terms of significantly upregulated genes in the comparison of Heat_N-/N-groups.

Table S8. Overrepresented GO terms of significantly downregulated genes in the comparison of Heat_N-/N-groups.

Table S9. Overrepresented GO terms of significantly upregulated genes in the comparison of Heat_N+/Heat_N- groups.

Table S10. Overrepresented GO terms of significantly downregulated genes in the comparison of Heat_N+/Heat_N-groups.

Table S11. Gene information in the weighted gene co-expression network. 